\title{
How I do it? A multimodality-guided awake hybrid operation for a language-area brain arteriovenous malformation and multiple intracranial aneurysms
}

\author{
Zixiao Yang ${ }^{1,2,3,4,5,6} \cdot$ Meng Deng $^{7} \cdot$ Yiheng Liu $^{7} \cdot$ Jianping Song ${ }^{1,2,3,4,5,8}$ (D)
}

Received: 22 September 2021 / Accepted: 16 December 2021 / Published online: 7 January 2022

(c) The Author(s), under exclusive licence to Springer-Verlag GmbH Austria, part of Springer Nature 2022

\begin{abstract}
Background The cure of an eloquent brain arteriovenous malformation (BAVM) and multiple intracranial aneurysms with preservation of neurological function and the minimal procedures is challenging.

Method A 53-year-old male was admitted to treat a left frontal language-area BAVM and concomitant five bilateral intracranial aneurysms. After repairing the ruptured right middle cerebral artery (MCA) bifurcation aneurysms and the other two unruptured ones, at the second-stage multimodality-guided awake hybrid operation, we successfully obliterated the left frontal BAVM and two other left MCA aneurysms.

Conclusion The multimodality-guided awake hybrid operation may be a promising technique to treat complicated cerebrovascular disease.
\end{abstract}

Keywords Awake surgery $\cdot$ Brain arteriovenous malformation $\cdot$ Hybrid operating room $\cdot$ Intracranial aneurysm

\section{Introduction}

A 53-year-old male presented with a headache for one day without neurological deficits. The emergent head computed tomography (CT) and CT angiography showed a right temporal hematoma caused by a right middle cerebral artery (MCA) aneurysm and a left frontal brain arteriovenous malformation (BAVM). The patient was then sent to the hybrid operation room for digital subtraction angiography (DSA) examination and prepared for emergent treatment. The DSA

This article is part of the Topical Collection on Vascular Neurosurgery - Arteriovenous malformation

Jianping Song

Neurosurgerysong@foxmail.com

1 Department of Neurosurgery, Huashan Hospital, Fudan University, No. 12 Wulumuqi Zhong Road, Shanghai 200040, China

2 National Center for Neurological Disorders, Shanghai 200040, China

3 Neurosurgical Institute of Fudan University, Shanghai 200040, China

4 Shanghai Clinical Medical Center of Neurosurgery, Shanghai 200040, China showed a large ruptured right MCA bifurcation aneurysm, a right anterior choroidal artery (AchoA) aneurysm, an anterior cerebral artery (ACA) A1 aneurysm, a left MCA bifurcation aneurysm, a left MCA M3 aneurysm, and a left frontal BAVM. The BAVM was supplied by the MCA and ACA and drained into the sagittal sinus (Fig. 1).

After evaluating the benefits and risks of microsurgical and endovascular treatment, we offered a two-stage hybrid surgery approach to obliterate all deadly lesions while making the utmost effort to protect the patient's brain function [8]. Informed consent has been obtained from the patient. First, the patient underwent microsurgery

5 Shanghai Key Laboratory of Brain Function Restoration and Neural Regeneration, Shanghai 200040, China

6 Research Units of New Technologies of Micro-Endoscopy Combination in Skull Base Surgery (2018RU008), Chinese Academy of Medical Sciences (CAMS), Shanghai 200040, China

7 Department of Anesthesiology, Huashan Hospital, Shanghai Medical College, Fudan University, Shanghai 200040, China

8 Department of Neurosurgery, Fudan University Huashan Hospital Fujian Campus, Fujian Medical University, The First Affiliated Hospital Binhai Campus, National Regional Medical Center, Fuzhou 350209, Fujian, China 
Fig. 1 The left internal carotid artery (LICA) angiography showed a left middle cerebral artery (MCA) bifurcation aneurysm, a left MCA M3 aneurysm, and a left frontal brain arteriovenous malformation (BAVM). The BAVM was supplied by the MCA and ACA and drained into the sagittal sinus

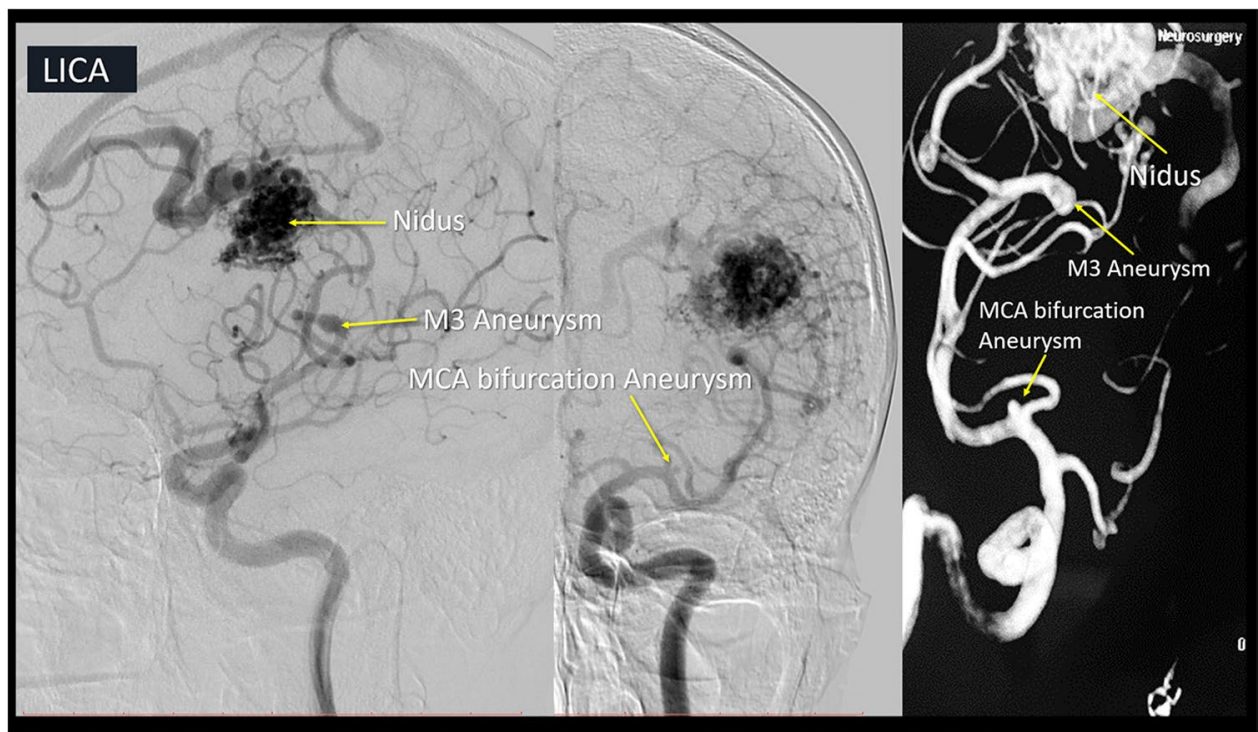

to repair the ruptured right MCA bifurcation, the right AchoA and ACA A1 aneurysms. Two months later, the second-stage hybrid surgery was scheduled, and multimodality-guided awake BAVM resection and left MCA bifurcation aneurysm and left MCA M3 aneurysm awake clipping were proposed. Given the sizeable volume of the maximal length of the BAVM at $3.2 \mathrm{~cm}$, the close relationship of the BAVM to the language area (Spetzler-Martin Grade III), and the deeply located ipsilateral concomitant aneurysms, second-stage surgery was deemed challenging.

\section{Relevant surgical anatomy}

Among the higher cortical functions, language is one of the most elaborate cognitive behaviors [6]. Classic models of language eloquent cortical areas posited that the motor and sensory language cortex existed in the Broca's area (pars opercularis and pars triangularis) and Wernicke's area, respectively [2]. However, cortical maps generated with intraoperative direct cortical stimulus (DCS) data showed surprising variability in language localization within the dominant hemisphere, and researchers have revealed that the eloquent language area is quite different from classical canonical classification [2, 7]. In this case, the preoperative blood oxygen level-dependent (BOLD) functional magnetic resonance imaging (fMRI) and diffusion tensor imaging (DTI) were conducted for eloquence assessment. The BOLD fMRI and the dorsal language pathway (arcuate fasciculus, AF, and superior longitudinal fasciculus, SLF) were reconstructed to show the relationship between the language eloquence and the BAVM (Fig. 2).

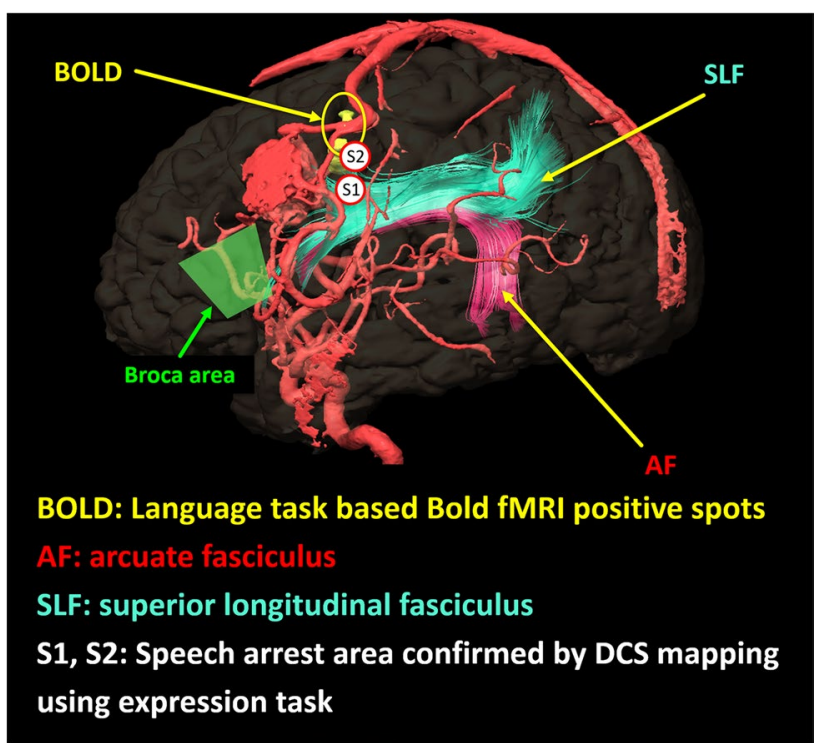

Fig. 2 This imaging showed the relationship of the BAVM nidus, the classic Broca area, the blood oxygen level-dependent (BOLD) functional magnetic resonance imaging (fMRI) activation area, and the dorsal language pathway (arcuate fasciculus, AF, and superior longitudinal fasciculus, SLF) tracked from diffusion tensor imaging (DTI). Intraoperatively, we did not locate the speech arrest area (S1, S2) in the classic Broca area's convexity surface or the BOLD fMRI activation area but located it in the premotor cortex posterior to the BAVM nidus that was the DTI SLF terminal territory in the mapping procedure

\section{Description of the technique}

We performed the multimodality-guided awake hybrid operation with a monitored anesthesia care technique [3, $8]$. The patient was placed in the supine position with a 
pterional approach. The intraoperative neuronavigation, electrophysiological monitoring system, and ultrasonography were used as essential tools.

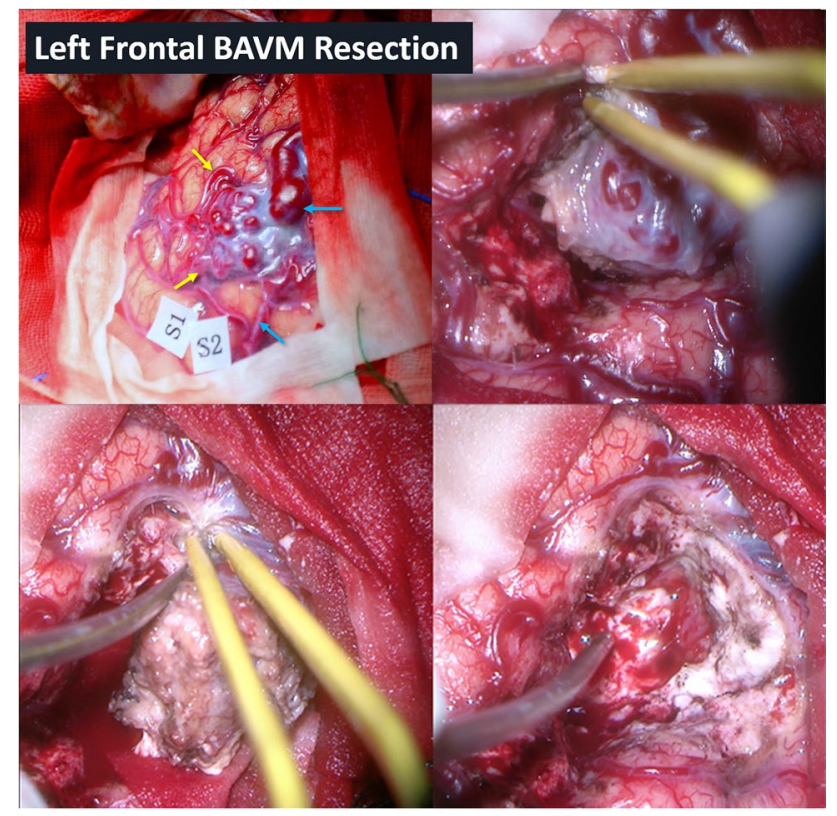

Fig. 3 After the patient awakened following the craniotomy procedure, we applied direct cortical stimulation to verify the speech output area (S1, S2) near the nidus. The BAVM was then carefully removed en bloc, and the eloquent gyrus was kept intact. Yellow arrow: feeding arteries of the BAVM nidus. Blue arrow: drainage veins of the BAVM nidus
First, the follow-up DSA in the hybrid room confirmed no recurrence of the right-side aneurysms, and 3D images of the left side vessels were used for intraoperative neuronavigation with fMRI and DTI (Fig. 3). After the patient awakened following the craniotomy, we applied DCS to verify the speech output area near the nidus. Patient fatigue may become an issue due to the extended awakening period to cure the BAVM and concomitant ipsilateral aneurysms. Therefore, we primarily applied the expression task using number counting in the DCS procedure to simplify cortical mapping. We did not locate the speech arrest area in the classic Broca area's convexity surface or the BOLD fMRI activation area but located it in the premotor cortex posterior to the BAVM nidus that was the DTI SLF terminal territory in the mapping procedure (Figs. 2, 3) [4]. We confirmed no abnormal vessel invasion to the eloquent gyrus under its cortical surface using ultrasonography. The BAVM was then carefully removed en bloc, and the eloquent gyrus was kept intact (Fig. 3). Then, since the left M1 is short and the M3 aneurysm was hidden deep inside, we opened the Sylvian fissure completely while being careful to protect the pia of the pars opercularis (classic Broca area). The patient was also asked to count numbers and answer questions during the clipping procedure. The MCA bifurcation aneurysm was secured first, and then, the M3 segment aneurysm was dissected and secured after short temporary occlusion (Fig. 4). Intraoperative angiography showed total obliteration of the lesions (Fig. 5). No language dysfunction was observed during the whole surgery, and the patient recovered well

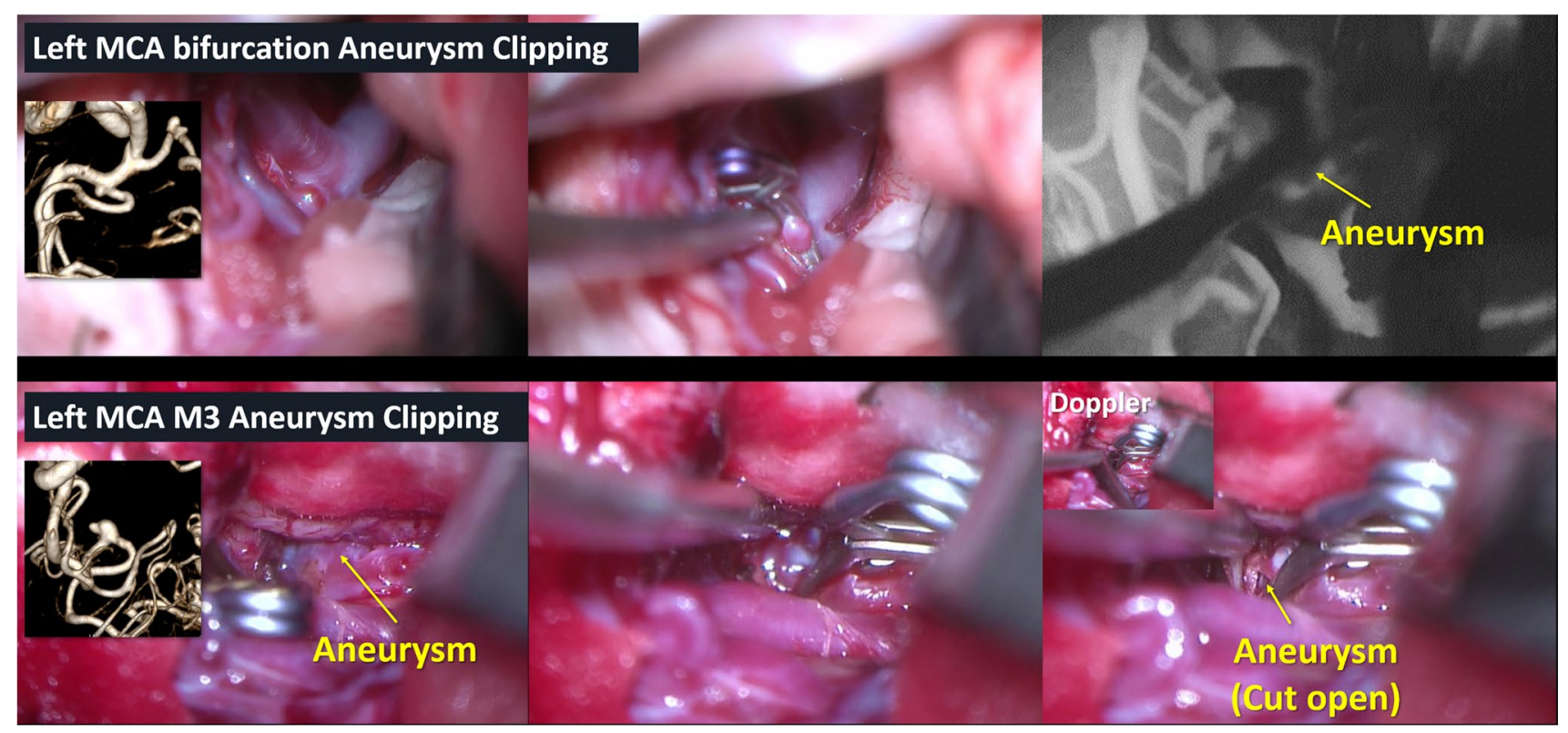

Fig. 4 Since the left M1 is short and the M3 aneurysm was hidden deep inside, we opened the Sylvian fissure completely while being careful to protect the pia of the pars opercularis (classic Broca area).
The MCA bifurcation aneurysm was secured first and confirmed by indocyanine green video angiography, and then the M3 segment aneurysm was dissected and secured after short temporary occlusion 


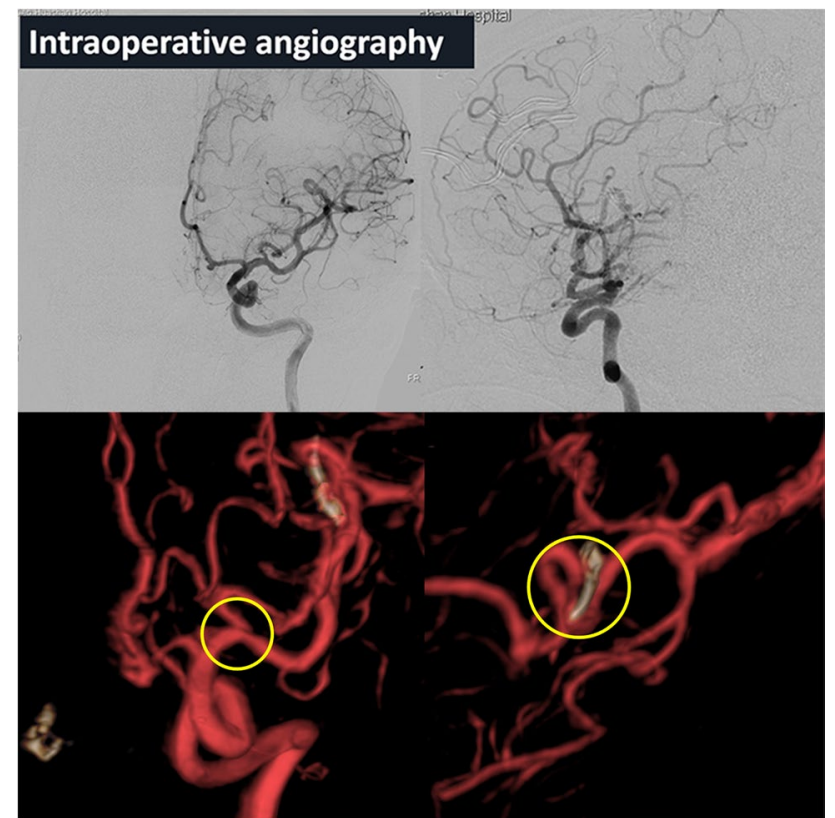

Fig. 5 Intraoperative angiography showed total obliteration of the lesions

postoperatively with transient epilepsy and aphasia. He fully recovered at the 1-month follow-up without any neurological deficits.

\section{Indications}

For this case, in consideration of the disease burden, the inevitable multistage endovascular treatment due to material supply shortages during the COVID-19 pandemic, and the patient's concern for a possible high recurrence rate after receiving endovascular treatment, we believe that two-stage hybrid surgery is the best way to cure patients with a minimal number of procedures. Since the left MCA aneurysms and the BAVM shared the same parent artery, the postoperative enlargement or even rupture risk of the aneurysms might be increasing due to the venous outflow occlusion after the nidus was removed. The MCA aneurysm was also considered to be best secured by clipping. Therefore, we decided to treat the BAVM and the ipsilateral aneurysms together for a cure in the second-stage surgery.

\section{Limitations}

The fMRI's most staggering limitation has been language lateralization, with low sensitivity (only $22 \%$ in naming tasks and $36 \%$ in verb generation tasks). Studies also have indicated that BAVM hemodynamics could impede BOLD signaling leading to inaccurate activation due to lesioninduced neurovascular uncoupling $[1,5,9]$. However, the neurofiber tracked based on DTI may not be influenced by the BAVM hemodynamic environment, like this case.

\section{How to avoid complications and specific perioperative considerations}

The risks of the procedure include the potential premature rupture of contralateral lesions after the first surgery, although the rupture risk was low. Second, the risk of awake anesthesia was not overlooked. Since the intracranial pressure cannot be controlled using the mechanical hyperventilation technique in awake surgery, we decided to remove the BAVM first to create more space for dynamic retraction while dissecting the Sylvian fissure and validate hemostasis.

\section{Specific information to give to the patient about surgery and potential risks}

The patient should be fully informed about the risks and benefits of the surgery. The particular concern includes the risk of premature rupture of the BAVM and aneurysms before or during the operation.

Supplementary Information The online version contains supplementary material available at https://doi.org/10.1007/s00701-021-05093-7.

Funding This study was sponsored by the Shanghai Rising-Star Program (18QA1400900) and the CAMS Innovation Fund for Medical Sciences (CIFMS, 2019-I2M-5-008).

\section{Declarations}

Research involving human participants All procedures performed in studies involving human participants were in accordance with the ethical standards of the institutional research committee and with the 1964 Helsinki Declaration and its later amendments or comparable ethical standards. The study was approved by Huashan Hospital Institutional Review Board (HIRB), Fudan University, Shanghai, China.

Informed consent It represents a video of a surgical case. The patient gave approval for this publication.

Conflict of interest The authors declare no competing interests.

\section{References}

1. Alkadhi H, Kollias SS, Crelier GR, Golay X, Hepp-Reymond MC, Valavanis A (2000) Plasticity of the human motor cortex in patients with arteriovenous malformations: a functional MR imaging study. AJNR Am J Neuroradiol 21:1423-1433 
2. Chang EF, Raygor KP, Berger MS (2015) Contemporary model of language organization: an overview for neurosurgeons. J Neurosurg 122:250-261

3. Deng M, Tu MY, Liu YH, Hu XB, Zhang T, Wu JS, Wang YW (2020) Comparing two airway management strategies for moderately sedated patients undergoing awake craniotomy: a single-blinded randomized controlled trial. Acta Anaesthesiol Scand 64(10): 1414-1421

4. Jeltema HR, Ohlerth AK, de Wit A, Wagemakers M, Rofes A, Bastiaanse R, Drost G (2020) Comparing navigated transcranial magnetic stimulation mapping and "gold standard" direct cortical stimulation mapping in neurosurgery: a systematic review. Neurosurg Rev 44(4):1903-1920

5. Lin F, Wu J, Jiao Y, Cai J, Cao Y, Wang S, Lin Y (2019) One-stage surgical resection of giant intracranial arteriovenous malformations in selected patients: a novel diffusion tenser imaging score. World Neurosurg 130:e1041-e1050

6. Mascitelli JR, Yoon S, Cole TS, Kim H, Lawton MT (2018) Does eloquence subtype influence outcome following arteriovenous malformation surgery? J Neurosurg 131:876-883

7. Sanai N, Mirzadeh Z, Berger MS (2008) Functional outcome after language mapping for glioma resection. N Engl J Med 358:18-27

8. Song J, Li P, Tian Y, An Q, Liu Y, Yang Z, Chen L, Quan K, Gu Y, Ni W, Zhu W, Mao Y (2020) One-stage treatment in a hybrid operation room to cure brain arteriovenous malformation: a single-center experience. World Neurosurg 147:e85-e97

9. Ulmer JL, Hacein-Bey L, Mathews VP, Mueller WM, DeYoe EA, Prost RW, Meyer GA, Krouwer HG, Schmainda KM (2004) Lesion-induced pseudo-dominance at functional magnetic resonance imaging: implications for preoperative assessments. Neurosurgery 55:569-579; discussion 580-561
Key Points 1. The surgical treatment of an eloquent BAVM and concomitant multiple intracranial aneurysms is challenging.

2. Language is one of the most elaborate cognitive behaviors among the higher cortical functions.

3. The preoperative and intraoperative of the eloquence assessment is important for BAVM resection.

4. The limitation of BOLD fMRI in cerebral vascular disease should be acknowledged.

5. Intraoperative DCS mapping is still the "gold standard" for locating language area in awake surgery.

6. The ultrasonography is useful for BAVM nidus boundary inspection.

7. In this case, since the intracranial pressure cannot be controlled using the mechanical hyperventilation technique in awake surgery, the BAVM can be removed first to create more space for dynamic retraction while dissecting the Sylvian fissure.

8. In case of a short M1 and the need of deep-seated M3, the Sylvian fissure should be opened completely for aneurysm exposure.

9. Intraoperative DSA in a hybrid operation room is helpful to confirm the surgical results immediately.

10. The multimodality-guided awake hybrid operation is a promising technique to treat complicated cerebrovascular disease.

Publisher's note Springer Nature remains neutral with regard to jurisdictional claims in published maps and institutional affiliations. 